\title{
Stress Biomarkers as Outcomes for HIV+ Prevention: Participation, Feasibility and Findings Among HIV+ Latina and African American Mothers
}

\author{
Dorie A. Glover - Elena F. Garcia-Aracena • \\ Patricia Lester · Eric Rice . \\ Mary Jane Rothram-Borus
}

Published online: 7 April 2009

(C) The Author(s) 2009. This article is published with open access at Springerlink.com

\begin{abstract}
Mothers living with HIV (MLH) are at high risk for acute and chronic stress, given challenges related to their HIV status, ethnicity, economic and urban living conditions. Biomarkers combined into a composite index show promise in quantifying psychosocial stress in healthy people, but have not yet been examined among MLH. According, we examined potential biomarker correlates of stress [cortisol and catecholamines from home-collected urine and basic health indicators (blood pressure, height and weight, waist-to-hip ratio) measured during an interview] among 100 poor African American and Latina mothers MLH and demographic-matched control mothers without HIV $(n=50)$. Participants had been enrolled in a randomized controlled trial about 18 months earlier and had either received (MLH-I) or were awaiting (MLH-W) the psychosocial intervention. Participation was high, biomarkers were correctly collected for $93 \%$ of cases, and a complete composite biomarker index (CBI) calculated for 133 mothers (mean age $=42$ ). As predicted, MLH had a significantly higher CBI than controls, but there was no CBI difference across ethnicity or intervention group. CBI
\end{abstract}

\footnotetext{
D. A. Glover $(\bowtie)$

Semel Institute, Child Division, Department of Psychiatry and Biobehavioral Sciences, University of California, 760 Westwood Blvd., Los Angeles, CA 90024, USA

e-mail: dglover@mednet.ucla.edu

E. F. Garcia-Aracena - P. Lester

Semel Institute, Child Division, Department of Psychiatry and Biobehavioral Sciences, UCLA School of Medicine, University of California, 760 Westwood Blvd.,

Los Angeles, CA 90024, USA

E. Rice $\cdot$ M. J. Rothram-Borus

Center for Community Health, Psychiatry and Biobehavioral

Sciences, University of California, Los Angeles, CA, USA
}

predicted CD4 counts independently after controlling for age, years since diagnosis, prior CD4 counts, medication adherence, and depression symptoms. The study demonstrates acceptability, feasibility and potential utility of community-based biomarker collections in evaluating individual differences in psychosocial stress.

Keywords Stress $\cdot$ Biomarkers $\cdot$ Female $\cdot$ Mental health . Ethnicity $\cdot$ Allostatic load

\section{Introduction}

Psychosocial stress correlates strongly with mental health symptoms, risky health practices and lifestyles (e.g., substance use, unprotected sex, poor medication adherence, sleep, diet, physical activity) and compromised physiological function-all factors that lead to increased risk for opportunistic infection and rapid HIV disease progression. With mapping of the human genome and technological advances in biological measurement, there is increased interest in the use of non-invasive biomarkers to quantify psychosocial stress. As articulated by Kraemer et al. (2002), biomarkers may be sought in order to (1) more accurately or precisely measure an event or process; (2) measure an event or process earlier in time, when the information may improve prevention and intervention opportunities; and (3) provide insights into the cause, prevention, course and treatment of a disorder that the diagnosis itself would not reveal (pp. 655). Studies among primarily healthy (and HIV negative) groups have shown success in using stress-influenced biomarkers to predict later physical and cognitive decline (Seeman et al. 2008), reflect the presence and severity of mental health symptoms (Glover and Stuber 2006) and estimate the size of the 
hippocampus, a brain structure highly influenced by acute and chronic stress (Glover et al. 2008).

Within the HIV research community, there is consensus that biological indicators can serve as mediators and moderators in precipitating symptoms of anxiety and depression, can influence immune system functioning, and may also alter the severity and progression of the disease (Kopinsky et al. 2004). Emerging studies of specific biomarkers in HIV+ samples confirm the relevance of stressinfluenced biomarkers in basic molecular mechanisms of HIVAIDS (Kopinsky et al. 2004; Kumar et al. 2003; Sherman et al. 2004). For example, norepinephrine (NE), a neurotransmitter of the autonomic nervous system (ANS) released in response to stress, has been shown to accelerate HIV-replication (Cole et al. 1998, 2003; Cole 2008). Novel interventions focusing on altering ANS activity generally, or NE specifically, may delay the development of clinical disease. Concurrently, promoting stress management among HIV+ persons has shown that reducing general distress, anxiety, and depression may alter immune and endocrine biomarkers among Caucasian men, as well as progression of HIV (Antoni 2003; Leserman 2003; McCain et al. 2003; Sherman et al. 2004).

Interventions that reduce psychological distress among HIV+ persons do not influence biological markers or do so inconsistently (Brown and Vanable 2008; Crepaz et al. 2008; Scott-Sheldon et al. 2008). The negative findings have been attributed to socio-demographic factors (age, gender, race/ethnicity), assessment timing (shortly after the intervention vs. months later), and analytic complexities of disease variables which themselves are known to be associated with dysregulated mood, cognition, and somatic and neurovegetative symptoms (stage of HIV, time since infection, medications). Biological systems are further influenced by health behaviors (sleep, diet, and level of physical activity), hormonal cycles (menses and menopause) and licit and illicit substances (tobacco, alcohol and drugs) (Chander et al. 2006; Kudielka and Kirschbaum 2005; Kirschbaum et al. 1999). Existing biomarker studies in HIV have sometimes been limited by incomplete attention to such potential mediators and moderators (Arendt 2006; Boarts et al. 2006; Kopinsky et al. 2004).

The selection of biomarker outcomes may also contribute to weak or equivocal biomarker effects in HIV intervention studies. Psychoneuroimmunological research typically requires drawing blood and processing samples on the same day with sophisticated assay techniques. The cost and practical barriers of this approach in communitybased research not only reduces the amount of research possible, but may also limit participation due to high subject burden. Also, most studies to date have focused on one or two systems/biomarkers-for example, a disease specific immune system indicator (CD4 counts), the ANS (norepinephrine) or the hypothalamic-pituitary-adrenal (HPA) axis (cortisol). These biomarkers are often examined in isolation, precluding a comprehensive understanding of interactions within and across biomarkers and systems.

An alternative approach is one first used within the context of epidemiological studies of healthy aging, based on the allostatic load (AL) model (McEwen 2004; McEwen and Seeman 1999; McEwen and Wingfield 2003). Briefly, the model describes the normal biological response to a stress perturbation as a series of adaptive changes in multiple systems designed to meet the challenge and then return to physiological stability or allostasis. Successful adaptation to stress is operationalized as the ability to maintain homeostasis in the face of internal and external perturbations. AL represents the cumulative price the body pays for adapting to repeated adverse psychosocial, physical, and environmental challenges. It is the inability to achieve and maintain allostasis that is thought to serve as the basis for long-term negative physical health consequences of stress. This framework uses a set of biomarkers combined into a composite index to quantify cumulative stress exposure.

A composite biomarker index of AL reflecting cardiovascular activity, atherosclerosis development, HPA axis functioning, glucose metabolism, and sympathetic nervous system (SNS) activation has been shown to predict cognitive and physical functioning, cardiovascular disease, and mortality in healthy elderly samples (Karlamangla et al. 2002; Seeman et al. 2001). The index also increases with age (Crimmins et al. 2003), is differentially higher among African American women across all ages (Geronimus et al. 2006), and is associated with posttraumatic stress symptoms (PSS) in mothers of children with a serious illness (Glover and Stuber 2006). In a study of illness-precipitated PSS among mothers of children with cancer, the index has been shown to discriminate between groups in a "doseresponse" manner (highest to lowest: PSS-symptomatic cancer mothers, non-symptomatic but chronically stressed cancer mothers, control mothers of healthy children), even when individual biomarkers do not (Glover and Stuber 2006). Recently, a high biomarker composite index was linked to smaller hippocampal volume in mothers of seriously ill children (Glover et al. 2008).

The composite biomarker approach offers several potential advantages for biomarker research among individuals with HIV. First, the biomarkers typically collected are non-invasive and relatively inexpensive, comprised from home-collected urine (cortisol and catecholamines), saliva (cortisol, DHEA), and brief general health measures familiar to participants (blood pressure, heart rate, height and weight). Second, the biomarkers represent multiple biological systems that can be analyzed individually, 
collectively, or in subsets to understand interrelationships across systems. Third, the approach uses relative risk composite scores that may be better predictors of poor health outcomes than levels of individual biomarkers that fall into a "clinical" range for pathology (Glover and Stuber 2006; Glover et al. 2008; Kopinsky et al. 2004). Composites inherently select "extreme" scorers, thereby supporting comparisons of individuals at greatest risk and those displaying the greatest "resilience" to HIV+ stress. Composite risk scores appear to offer maximal sensitivity to potentially small-to-moderate effects as might be seen in community-based intervention samples.

On the other hand, composite risk scoring methods to date are based on a primary assumption that is increasingly questioned for population studies and may be especially unlikely to hold true in HIV+ samples. Specifically, scoring confers a risk "point" for each biomarker in the top quartile of the sample distribution and the direction of risk is assumed to be unidirectional. Thus, high levels of primary neuroendocrine mediators (cortisol and catecholamines) as well as secondary cardiovascular (blood pressure, heart rate) and metabolic (body mass index, waist to hip ratio) outcomes are assumed to be reflective of risk. There is now substantial evidence for bidirectional risk (too high or too low) in primary mediators. For example, despite extensive data showing detrimental health effects of high cortisol, numerous studies have now shown low cortisol in PSS samples (Glover and Stuber 2006; Yehuda et al. 1996) and in elderly individuals with high depression, perceived stress, and physical complaints (Hellhammer et al. 2004). Equivocal directionality of other primary neuroendocrine biomarkers and secondary outcomes is cited as the rationale for scoring all biomarkers with bidirectional risk (Glei et al. 2007; Seplaki et al. 2005). Such a strategy seems warranted in HIV+ samples, where PSS symptoms may be prevalent (Gaynes et al. 2008; Israelski et al. 2007; Myers et al. 2006; Whetten et al. 2006, 2008) and lipodystrophy or other metabolic disturbances are known sequelae of $\mathrm{HIV}+$ infection, antiviral medications, or both (Chang et al. 2007).

To our knowledge, a composite biomarker index has never been reported for HIV+ samples. Accordingly, in this study we examine a bi-directional biomarker risk composite in relation to effects of ethnicity (African American and Latina) and HIV status (HIV+ or HIV-) among low-income minority women at high risk for psychological stress. Women of racial and ethnic minority backgrounds are the fastest growing proportion of new AIDS diagnoses in the United States. According to recent numbers from the Centers for Disease Control and Prevention [CDC] (2009), African American and Latina women represent $82 \%$ of the total estimated AIDS diagnoses. AIDS is the leading cause of death among African American (AA) women ages 25-34. For Latinas (LA), AIDS is the fourth leading cause of death among women 35-44. Our sample is a subset recruited from a larger intervention study for mothers living with HIV (MLH) and their children (described by Rotheram-Borus et al. 2001). The sample consists of MLH who received a psychosocial intervention (MLH-I) and those awaiting the intervention (MLH-W).

A necessary step in examining the potential utility of stress-related biomarker composites in HIV-related intervention research is to establish acceptability (participation) and feasibility (accurate completion of collection protocols within low-resource settings). If these aims are met, our additional goal is to investigate the utility of a biomarker composite to differentiate groups on the basis of HIV (MLH vs. controls), ethnicity (AA vs. LA) or intervention status (MLH-I vs. MLH-W). Reflecting the burden of HIV on mental and physical health outcomes, we predict MLH groups will show higher biological risk scores than HIVcontrol mothers. We make no predictions for ethnicity, but sample for an equal number of AA and LA women in each group to examine possible ethnicity effects. Finally, we predict that MLH-I will show lower biological risk scores than MLH-W awaiting the intervention. Analyses of the larger dataset to date show no effect of the intervention on mother self-report psychosocial stress measures, perhaps because baseline MLH scores indicate relatively low psychological distress (S. Comulada, personal communication, March 3, 2009). Nonetheless, biological risk scores may be more sensitive to group effects than self-report measures.

\section{Methods}

\section{Participants}

MLH were recruited from a larger parent study of a familybased HIV prevention and coping intervention in Los Angeles County (Rotheram-Borus et al. 2001). From January 2005 to October 2006, 342 women living with HIV+ who were living in poverty and the mother/primary female caregiver of a child between the ages of 6 and 20 years old were recruited from HIV clinics, health clinics, women's support group and advertising (refusal rate $=12.5 \%$ ). Concurrently, 205 HIV - mothers were recruited from public venues (e.g., outside grocery stores) in the same neighborhoods where MLH lived. HIV - mothers were matched to MLH based on race/ethnicity, age of children, and neighborhood of residence. All mothers received a full battery of standardized measures of psychological and physical health at 6 month intervals. Most families lived below the poverty line (\$20,000) (86\% of MLH and $66 \%$ of 
controls). Illicit drugs were used by only a small minority of mothers and were not the source of HIV infection. MLH were randomly assigned to an immediate intervention (MLH-I) or wait list (MLH-W) which offered the intervention at a later time. The basic eight-session intervention focused on coping with HIV and being a parent. Up to 8 additional booster sessions were also offered for a total of 16 possible intervention sessions.

A subset of MLH $(n=100)$ and controls $(n=50)$ from this larger cohort were recruited for the biomarker assessment and MLH were composed of 50 each of MLH-I and MLH-W. Recruitment aimed for an equal number of African American and Latina women in each group to allow for ethnic comparisons as recommended (ScottSheldon et al. 2008). Biomarker assessment was timed to coincide with the ongoing 18 or 24-month follow-up interviews of the parent study. The biomarker project was explained and offered as a separate study to each consecutive MLH and control scheduled for a follow-up interview until the sample goal of $N=150$ was met.

\section{Measures}

The biomarker index was created from home-collected urine (cortisol and catecholamines) and standard health measures (blood pressure, heart rate, height and weight) (see Table 1.) Urinary indicators were collected at home by participants during a 12-h overnight collection period designed to represent "resting" hormone values. Standard health measures were recorded during a scheduled interview conducted within a few days of the urine collection. Heart rate and systolic and diastolic blood pressure were measured using an automatic inflation digital device meeting the Association for Advancement of Medical Instrumentation (AAMI) standards and with appropriate cuff size, following standard protocol (Pickering 1996). Supplies for urine collection (a urine specimen container, toilet "hat" and cooler) were provided.
Urine was collected in a container with a preservative ( $0.3 \mathrm{~g}$ of sodium metabisulfite). Samples were kept cool in an "igloo" container until transport to the laboratory. After evaluation of total urine volume, aliquots were frozen at $-80^{\circ} \mathrm{C}$ until analyzed via EIA and Enzyme Immunoassay for the quantitative determination of endocrine concentration (cortisol and catecholamines). Safeguards for assuring compliance to urine collection procedures include measuring the volume of urine, the amount of creatinine per total volume, and the concentration of each biomarker in total and as a function of the creatinine level. There are reference ranges for each of these measurements that allow for identification of potentially contaminated samples, which were discarded according to universal precautions.

Risk was calculated as bi-directional, as recommended (Glei et al. 2007; Seplaki et al. 2005). The biomarker index was calculated by assigning one risk "point" for each biomarker falling in the bottom (15th percentile) or top (85th percentile) of the total sample distribution and then summing the risk points. Each participant could therefore score between zero and nine, reflecting the total possible number of biomarkers in a "high-risk" range (either very high or very low).

Existing data on sociodemographics, health status, and medication usage in HIV+ mothers were collected from the parent study. New instruments for the biomarker supplement consisted of three brief instruments. The Health Behaviors Questionnaire (HBQ) is a short self-report questionnaire to assess activities in the $24 \mathrm{~h}$ prior to biomarker collection. Questions monitor caffeine drinks, cigarettes, alcoholic beverages (number of), aerobic and anaerobic exercise (minutes and quality), sleep (hours and quality), meals (number and timing), prescribed and recreational medication (type and amount), and illness symptoms (fever, cold, flu, headache, etc.). Information from the HBQ was used to exclude urine samples that may be contaminated by known confounders and to identify potential associations with the biomarker index. The

Table 1 Summary of biomarkers

\begin{tabular}{|c|c|}
\hline & Procedures \\
\hline Diastolic BP (mm Hg) & Average of second and third of 3 seated readings at rest and after habituation to environmen \\
\hline \multicolumn{2}{|l|}{ Systolic BP (mm Hg) } \\
\hline \multicolumn{2}{|l|}{ Heart rate $(\mathrm{bpm})$} \\
\hline Waist to hip ratio & $\begin{array}{l}\text { Waist circumference measured at its narrowest point between the ribs and iliac crest and hip } \\
\text { circumference measured at the maximal buttocks }\end{array}$ \\
\hline Body mass index & $703 \times\left[\right.$ Weight in pounds/height $\left.{ }^{2}\right]$ Am Heart and Lung Institute \\
\hline Norepinephrine $(\mu \mathrm{g} / 12 \mathrm{~h})$ & Assayed from 12-h overnight urine collection. Calculated as total concentration in 12-h urine \\
\hline Epinephrine $(\mu \mathrm{g} / 12 \mathrm{~h})$ & volume and as portion per creatinine level (See Glover and Poland 2002) \\
\hline \multicolumn{2}{|l|}{ Dopamine $(\mu \mathrm{g} / 12 \mathrm{~h})$} \\
\hline Cortisol $(\mu \mathrm{g} / 12 \mathrm{~h})$ & \\
\hline
\end{tabular}


Posttraumatic Stress Scale (PSS) (Foa et al. 1997) uses DSM-IV criteria to evaluate Criterion A traumatic exposure history and symptom clusters of re-experiencing, avoidance/numbing, and hyperarousal. The PSS is a common research instrument that compares well to clinician ratings obtained in structured interviews. The Beck Depression Inventory (BDI) is a 21-item self-report questionnaire widely used to assess symptoms of depression (Beck et al. 1988). All instruments were completed in the language preferred by the participant (English or Spanish).

\section{Procedures}

All participants of the intervention study who were about to be scheduled for their regular follow-up interviews were informed of the biomarker pilot and asked to participate. Recruitment continued until the total sample (balanced for ethnicity) was consented. For those consenting, existing staff then conducted a brief interview, collected partial biomarker data (diastolic and systolic blood pressure, height and weight, and hip to waist ratio) and gave instructions and supplies needed for the 12-h urine collection and a later saliva collection. The interview also included administration of the PSS and BDI. (Results from home-collected saliva assayed for cortisol and DHEA-S are reported elsewhere: Glover et al. 2009). This study was approved by the UCLA Institutional Review Board.

Participants were instructed to begin to collect urine near bedtime and end urine collection right after their customary waking time (including the first morning void) so as to collect all voids within a $12 \mathrm{~h}$ period. Instructions for urine sample included (1) avoiding contaminants in urine (rescheduling collection if menstruating or sexual intercourse occurred during the collection); (2) rescheduling if transitory illness occurred (cold/flu); (3) abstaining from substance use (other than prescribed medications). The HBQ was reviewed in advance to inform participants of what could unduly influence urine collection and impede correct interpretation of assays. Participants completed the HBQ after biomarker collection and data were reviewed for verification and to analyze the potential impact of specific behaviors on the biomarker index. Participants were asked to refrain from non-prescribed substance use (alcohol and drugs) for $24 \mathrm{~h}$ prior to biological sample collection and to re-schedule the collection if they were feeling ill, if they had an unusual or atypical day, etc. We explained that the biomarkers are used to assess their own overall health and that the accuracy of the measurement depends on their ability to follow instructions in collection and procedures. They were encouraged to remain on their prescribed medications (including HIV medications) as directed by their physicians.
Statistical Analysis

Percentiles for specific biomarker indicators were identified and one risk point assigned for each indicator falling in the range of risk (highest or lowest for the total sample). Risk points were summed and frequency of risk for each biomarker as a function of group was calculated. The final composite index was examined for normality and with Levine tests to examine violations in homogeneity of variance before conducting Analysis of Covariance (ANCOVA). ANCOVA procedures were also used to compare raw values of each indicator across groups after controlling for age. Linear regression methods were used to examine the predictive value of the composite for most recent $\mathrm{CD} 4$ counts.

\section{Results}

Acceptability and Feasibility

Only three potential participants from the larger cohort refused the present study. Complete biomarker collections and interviews were conducted with a total of 147 participants. Of these, eight $(\mathrm{MLH}=4$, Controls $=4)$, were discarded due to urine volumes or creatinine levels indicative of error in collection (volume lower than expected or creatinine values out of the normative range). Three more cases were incomplete, with two missing systolic and diastolic blood pressure due to a mechanical failure of the machine and one with an undetectable level of norepinephrine in urine. Biomarkers were correctly collected for 136 (93\% of the total). Self-reported recreational drug use was rare in this sample. Six participants reported marijuana use within $24 \mathrm{~h}$ of biomarker collection (2 AA controls, 3 AA MLH-W and 1 Latina MLH-I). Repeating analyses without these cases did not alter the biomarker index findings. One participant reported use of crack cocaine and two reported more than three alcoholic drinks during the period. These three cases were dropped from analysis. The final sample $(N=133)$ consisted of Controls $(n=44)$ and $\operatorname{MLH}(n=89)$.

\section{Health Behaviors}

Groups did not differ on number of caffeinated $(M=1.45$, $\mathrm{SD}=1.8)$ or alcoholic drinks $(M=0.09, \mathrm{SD}=0.4)$, cigarettes $(M=2.21, \quad \mathrm{SD}=5.3)$, minutes of aerobic $(M=7.37, \quad \mathrm{SD}=24.9) \quad$ or $\quad$ anaerobic $\quad(M=21.60$ $\mathrm{SD}=41.4)$ exercise or hours of sleep on the night of urine collection $(M=7.04, \mathrm{SD}=2.1)$. None of these variables were related to the biomarker composite. 
Sociodemographics

Overall, MLH and controls did not significantly differ on age, ethnicity, education, or number of persons living in the home. Significantly more control mothers $(46.3 \%)$ reported having a job than MLH (27.9\%), and reported greater monthly income (Controls: $M=\$ 1,615, \mathrm{SD}=\$ 1,500$, range $=\$ 168-9,000)$ than $\mathrm{MLH}(M=\$ 990, \mathrm{SD}=\$ 730$, range $=0-\$ 9,000)$. Both groups reported they were struggling or barely able to pay their bills (Controls: 57.5\%; MLH: $47.8 \%$ ) relative to those reporting they were "doing ok" financially. The final sample had slightly more LA $(53.3 \%)$ than AA $(46.7 \%)$ mothers. Age was not significantly different by group or ethnicity, but a significant group $X$ ethnicity interaction $[F(1,133)=4.83, P<.05]$ indicated no ethnic differences in age among controls $(M=42.61, \mathrm{SD}=8.26)$, but older age among AA MLH $(M=45.14 \mathrm{SD}=7.9)$ than among LA MLH $(M=40.38$, $\mathrm{SD}=7.9)$. Education differed by ethnicity $\left[X^{2} \quad(1\right.$, $N=134)=25.53, P<.01] ; 76.4 \%$ of LA vs. $33.9 \%$ of AA reported less than a high school education. Also, LA mothers reported significantly more adults living in the home $(M=2.11, \mathrm{SD}=1.1)$ than AA mothers $(M=1.63$, $\mathrm{SD}=1.0$ ), but the groups did not differ on the reported number of children at home $(M=2.87, \mathrm{SD}=1.7)$. Most LA mothers $(83.6 \%)$ participated in assessments in Spanish (and conducted by Spanish-speaking interviewers). The biomarker composite was not associated with education, number of adults or children in the home or primary language.

\section{HIV Variables}

Among MLH, 97\% of self-reports of CD4 cells were over 200 in $97 \%(M=576.93, \mathrm{SD}=212.3$, range $0-1,400)$ and $87 \%$ reported undetectable viral loads. There were $66.3 \%$ $(n=61)$ who reported on antiviral medication adherence. Of these, $70.5 \% \quad(n=43)$ reported $100 \%$ medication adherence in the last 3 days, and the remaining $29.5 \%$ ( $n=18$ ) admitted skipping medications on one or more of the past 3 days. Viral load, CD4 cell count, and medication adherence were not significantly different as a function of ethnicity or intervention status. MLH reported learning of their HIV status from 2 to 25 years ago $(M=11.57$, $\mathrm{SD}=5.0$ ) with two cases reporting "do not know". AA MLH had known their HIV status longer than LA MLH. The biomarker composite was not significantly correlated with time since infection, viral load or baseline CD4 counts, but was negatively correlated with most recent CD4 counts (Pearson $r=-.26, P<.05$ ). All biomarker composite analyses included age, and intervention analyses (excluding controls) also included years since HIV diagnosis.
Intervention Participation (MLH-I Only)

Of 48 MLH-I offered the psychosocial intervention 6 (12.5\%) did not complete any sessions (4 AA MLH and 2 LA MLH). Of the remaining $42(87.5 \%)$, the mean number of sessions attended was 18.1 ( $\mathrm{SD}=5.7)$, with a range of 9-36. The number of sessions attended was not significantly related to any socio-demographic, health behavior, HIV or psychological variables or the biomarker composite itself.

Psychological Variables

ANOVA indicated a significant group effect for BDI scores $[F(2,131)=3.62, P<.05]$ and planned contrasts indicated MLH-I $(M=12.42, \quad$ SE $=1.4)$ and MLH-L $(M=13.57, \quad \mathrm{SE}=1.5) \quad$ reported significantly more depression than controls $(M=8.14, \mathrm{SE}=1.5)$. BSI global severity scores did not show a significant group effect. There was no evidence of ethnicity differences in depression or global distress. On the PSS, 37 participants said they had never experienced any of the potentially traumatic events listed, and another 23 who reported having at least one trauma exposure said the event did not bother them. Thus, there were 60 "non-responders" and 74 responders (who said they had experienced at least one traumatic event that bothered them and answered questions about symptoms). Non-responders were disproportionally control mothers (31 of 60 ); $70 \%$ of all controls but only $32 \%$ of MLH were non-responders. Among MLH, non-responders were significantly more depressed than responders and 12 of $61 \mathrm{MLH}$ with both BDI and PSS scores met criteria for clinically relevant levels of both depression (BDI $>16$ ) and PTSD (PSS $>18$ ) symptoms. PSS scores were unrelated to the biomarker composite. The composite index was significantly associated with depression $(r=.29, P<.05)$ and BSI global severity of distress $(r=.34, P<.05)$ for controls, but not for MLH $(r<.20)$. Among MLH, none of the subscales of the BSI correlated with the composite index.

\section{Individual Biomarkers}

As seen in Table 2, raw values for each of the biomarkers indicated no effect of group (control vs. MLH) or intervention (MLH-I vs. MLH-W). Significant ethnicity differences emerged for urinary cortisol and catecholamines and cardiovascular variables (blood pressure, heart rate). Among controls and MLH, AA mothers were elevated relative to LA mothers on all cardiovascular variables (systolic and diastolic blood pressure and resting heart rate: $P<.01)$. Cortisol was elevated in LA versus AA control mothers $[F(1,44)=10.76, P<.01]$. Among 


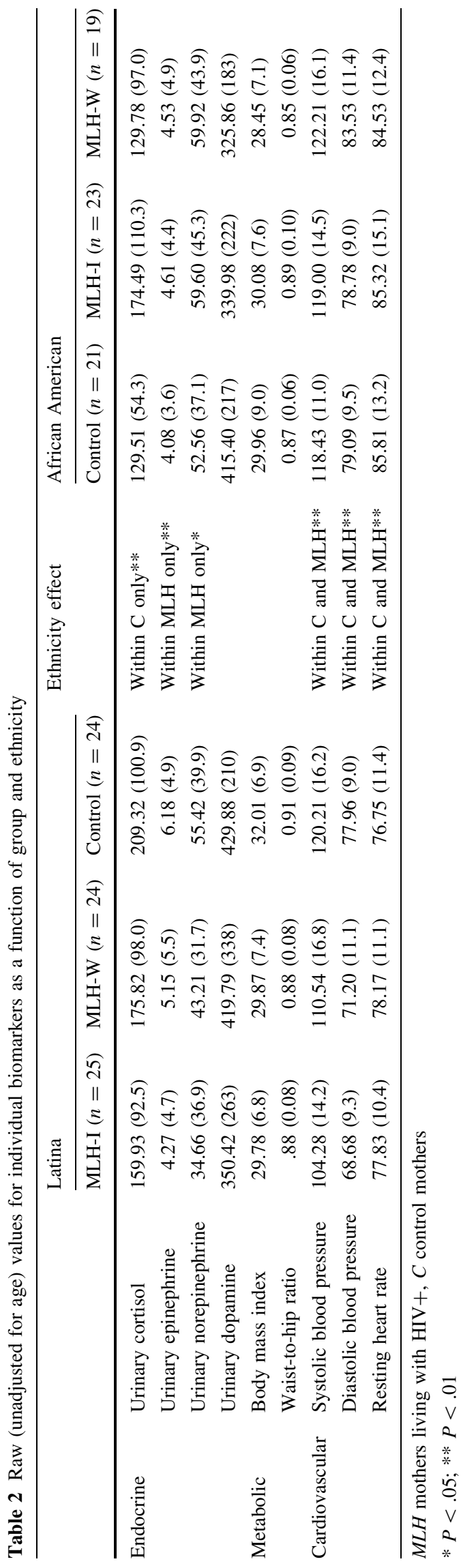

MLH only, AA had higher norepinephrine than LA mothers $[F(1,89)=6.26, P<.01]$, whereas LA mothers showed significantly higher epinephrine $[F(1,44)=4.77$, $P<.05]$. Chi-square analyses of bi-directional risk points (yes/no) for each biomarker indicated no significant group, intervention or ethnicity effects (not shown).

Composite Biomarker Index

As predicted, the ANCOVA indicated MLH had significantly higher biomarker composites $(M=2.54, \mathrm{SD}=1.4)$ than controls $(M=1.75, \mathrm{SD}=1.3)[F(1,133)=8.22$, $P<.001]$ and no ethnicity or group $\mathrm{X}$ ethnicity interaction. See Table 3 for the raw biomarker composite scores for all participants as a function of group and ethnicity.

To examine an intervention effect (MLH-I vs. MLH-W) in the biomarker composite, covariates added to the ANCOVA included: age, time since HIV infection, baseline CD4 counts, medication adherence (last 3 days yes/ no) and number of intervention sessions attended (analyzed by removal of the MLH-I with 0 attendance and by retaining them but including sessions attended as a covariate (coding MLH-W as 0 attendance). There was no significant intervention effect and medication adherence was the only significant covariate $(P<.04)$. A separate ANOVA indicated those MLH who reported adherence over the past 3 days had higher composite scores $(M=2.65, \quad \mathrm{SD}=1.4) \quad$ than non-adherent $\mathrm{MLH}$ $(M=1.90, \mathrm{SD}=1.37)$. Medication adherent MLH also reported lower CD4 counts, suggesting adherent MLH may be more ill than non-adherent MLH.

To determine if the biomarker composite provided further predictive value for most recent CD4 counts after controlling for other known predictors, a linear regression model was developed. Age, baseline CD4, medication adherence, psychological variables and group intervention status were entered first, followed by the biomarker composite. The PSS, BSI and group intervention status were not significant predictors and were dropped. As shown in Table 4, the overall model significantly predicts most recent CD4 counts, $R^{2}=.53, R^{2}$ adjusted $=.49, F(6$, $85)=14.69, P<.001$. Three of the seven predictor variables significantly contributed to the model: (1) baseline CD4 counts; (2) depression symptoms and (3) the biomarker composite. Baseline CD4 counts were positively associated with current CD4 levels, whereas both depression symptoms and the biomarker composite were negatively associated with current CD4 (as depression and biomarker composite scores increased, CD4 levels decreased).

To determine if the model was equally predictive for AA and LA mothers, the regression model was repeated separately for each ethnicity. The overall model remained 
Table 3 Raw (unadjusted for age) biomarker composite index as a function of group and ethnicity

\begin{tabular}{|c|c|c|c|}
\hline & AA $(n=61)$ & $\mathrm{AA}(n=72)$ & Total biomarker index \\
\hline \multirow[t]{2}{*}{ Controls $(n=44)$} & $1.55(0.9)$ & $1.92(1.6)$ & $1.75(1.33)$ \\
\hline & $N=20$ & $N=24$ & \\
\hline \multirow[t]{2}{*}{$\operatorname{MLH}(n=89)$} & $2.50(1.3)$ & $2.57(1.6)$ & $2.54(1.4)$ \\
\hline & $N=41$ & $N=48$ & \\
\hline \multirow[t]{2}{*}{ MLH-I $(n=47)$} & $2.61(1.3)$ & $2.29(1.6)$ & $2.45(1.5)$ \\
\hline & $N=23$ & $N=24$ & \\
\hline \multirow[t]{2}{*}{ MLH-W $(n=42)$} & $2.39(1.2)$ & $2.63(1.6)$ & $2.52(1.4)$ \\
\hline & $N=18$ & $N=24$ & \\
\hline Total biomarker index & $2.20(1.3)$ & $2.28(1.6)$ & $2.24(1.5)$ \\
\hline
\end{tabular}

$A A$ African American, LA Latina, MLH Mothers living with HIV+

Table 4 Regression of most recent CD4 count on age, HIV+ variables, psychological symptoms, intervention group and the biomarker composite

\begin{tabular}{|c|c|c|c|c|c|c|}
\hline & \multirow{2}{*}{$\begin{array}{l}\text { Unstandardized } \\
\text { regression coefficient }(B)\end{array}$} & \multirow[t]{2}{*}{ Standard error of $B$} & \multirow{2}{*}{$\begin{array}{l}\text { Standardized } \\
\text { regression Coefficient } \\
\text { (Beta) }\end{array}$} & \multirow[t]{2}{*}{$t$ Value } & \multicolumn{2}{|l|}{$t$ Value } \\
\hline & & & & & AA only & LA only \\
\hline Age & 2.00 & 2.20 & .08 & .91 & .35 & .89 \\
\hline Years since diagnosis & -1.75 & 3.54 & -.04 & -.49 & 1.54 & -1.66 \\
\hline Baseline CD4 & .56 & .07 & .63 & $7.86^{* * * *}$ & $4.52 * * *$ & $6.75 * * *$ \\
\hline Medication adherence (no/yes) & 49.81 & 41.22 & .10 & 1.21 & 1.06 & .03 \\
\hline Depression (BDI) & -4.59 & 1.68 & -.21 & $-2.73 * *$ & -.84 & $-3.04 * *$ \\
\hline Biomarker composite & -33.68 & 11.73 & -.23 & $-2.87 * *$ & $-2.40 *$ & $-2.17 *$ \\
\hline Constant & 361.66 & 105.54 & & 3.43 & 1.31 & $3.33 * *$ \\
\hline$R^{2}$ & .53 & & & & .54 & .70 \\
\hline Adjusted $R^{2}$ & $.49 * * *$ & & & & $.46^{* * *}$ & $.65 * * *$ \\
\hline
\end{tabular}

$A A$ African American, LA Latina

$* P<.05 ; * * P<.01 ; * * * P<.001$

significant for AA $F(6,39)=6.45, P<.001$ and for LA $F(6,45)=15.16, P<.001$, but differences based on ethnicity emerged in several variables. First, baseline CD4 levels and the biomarker composite remained significant predictors of current CD4 for AA and LA, but depression scores were only predictive for LA mothers. Second, the $t$-values for years since diagnosis were in opposite directions, although not significant for either ethnicity. This may reflect differential HIV infection progression as a function of ethnicity. Finally, the variance explained for LA was higher $\left(R^{2}=.65\right)$ than for AA $\left(R^{2}=.46\right)$.

\section{Discussion}

This first study of a composite biomarker index in low income, Latina and African American mothers with and without HIV indicates that MLH and HIV-controls found the biomarker collection procedures highly acceptable. The participation rate was high and over $90 \%$ of the mothers provided usable data. Thus, we demonstrate the acceptability of the multiple biomarker collection procedures among minority mothers and the feasibility of obtaining valid samples from these groups in low-resource settings. The utility of the biomarker composite to differentiate groups on the basis of HIV status (MLH vs. controls) was also demonstrated. As predicted, HIV - control mothers demonstrated a lower biomarker risk index than MLH. Contrary to predictions, MLH-I who had received an intervention were not significantly lower on biomarker composite scores than MLH-W awaiting the intervention. These findings are consistent with analyses to date from the parent study, indicating no effect of the intervention on traditional psychosocial stress measures (S. Comulada, personal communication, March 3, 2009) and extend the absence of MLH intervention effects to a biomarker composite index. No significant ethnicity effects were found for the index scores.

Replicating previous studies among healthy women (Glover and Stuber 2006; Glover et al. 2008), the present 
data show that the biomarker composite is associated with clinically relevant outcomes among HIV+ samples. Greater self-reported medication adherence was associated with a higher composite and lower CD4 counts, suggesting a potential role for the composite in understanding the impact of worsening illness symptoms on medication adherence. Furthermore, the composite independently predicted most recent CD4 counts after controlling for known predictor variables (age, years since diagnosis, prior baseline CD4 counts, medication adherence, and depression symptoms). Improving upon prior research, this study also examined general health behaviors and HIV-specific health variables that might serve as potential mediators or moderators of biomarker outcomes, as recommended (Arendt 2006; Boarts et al. 2006; Kopinsky et al. 2004). None of the self-reported health behaviors [sleep, diet, exercise, caffeine and tobacco use, or substance (alcohol and drug) use] were different in controls compared to MLH mothers, nor were these significantly correlated with the biomarker composite. However, these mothers were not regular substance users, and so composite results may be different for individuals with very poor health behaviors and/or concurrent substance abuse. HIV-specific factors (CD4, viral load, medication compliance, time since infection), as measured here, were also unrelated to the composite index directly. Small sample size may have been a factor, and it would be helpful to confirm the absence of health behavior effects in future studies.

Results here also extend prior findings of a biomarker composite's utility in discriminating groups even when analyses of specific biomarkers indicate no differences. MLH means for each individual biomarker were not significantly different than controls, despite data suggesting $\mathrm{HIV}+$ sample differences in cortisol, catecholamines, autonomic activity and lipid distribution (Chittiprol et al. 2008; Kemeny and Schedlowski 2007; Kumar et al. 2002, 2003). This is likely due in part to the complex bi-directionality of health risk, which cannot be detected when averaged scores are used. For example, elevated body mass index (BMI) and waist-to-hip ratio (WHR) are health risks among the general HIV - population, but body wasting and associated low BMI and WHR are important health risks for HIV+ samples. Also, elevated cortisol is sometimes associated with poor HIV outcomes (Antoni et al. 2000, 2005; Cruess et al. 2000; Ironson et al. 2002; Leserman 2003) but findings are inconsistent and mechanisms of cortisol dysregulation remain complex (Cole 2008; Sapolsky et al. 2007). The significant associations found here between the bi-directional composite and HIV variables (CD4 cell counts and medication adherence) are promising, future studies must confirm that a bi-directional biomarker composite is predictive of meaningful clinical outcomes and psychological symptoms. Beyond the bi-directionality of risk, combining multiple biomarkers into a composite risk score appears to offer predictive value that is absent with any one or a few indicators alone. Even the chi-square analyses of bi-directional risk points across groups did not detect HIV status, intervention or ethnicity differences for any single biomarker. These findings support the multisystem emphasis of the allostatic load framework (Korte et al. 2005).

The absence of a relationship between the biomarker composite and psychological symptoms, however, is incongruent with the concept of allostatic load and is contrary to previous data indicating a link with depression and posttraumatic stress symptoms (PSS) (Glover and Poland 2002; Glover et al. 2008; Glover and Stuber 2006). Although depression symptoms were associated with the biomarker composite among control mothers, PSS and global BSI psychological distress were not. Furthermore, none of the psychological measures were linked to the biomarker composite for MLH. Also, the final regression model for MLH showed that the biomarker composite was an independent predictor of most recent CD4 counts, accounting for unique variance after controlling for depression. If the composite represents the cumulative burden of stress as theorized (McEwen 2004; McEwen and Seeman 1999; McEwen and Wingfield 2003) one would expect the index to be associated with depression, global distress, and post-traumatic stress symptoms-among controls and MLH mothers.

Several explanations are possible. Past studies showing a biomarker composite-psychological symptom link are different from the present research in several ways. First, previous studies supportive of the AL model utilize unidirectional composite scoring. Although bi-directional composite scoring such as that used here has been recommended (Glei et al. 2007; Seplaki et al. 2005), this method has not yet been widely examined. Relatedly, because of the many potential bi-directional effects of $\mathrm{HIV}+$ and/or medications, future studies may require a different set of biological indicators to quantify psychosocial stress than those used in large population studies. Finally, ethnicity effects may have obscured psychological symptom impact on the biomarker composite. AA women (controls and MLH) had significantly higher blood pressure and heart rate levels than LA women, but small cell sizes $(n<25)$ limited power to detect ethnicity and group X ethnicity interactions. Nonetheless, the final regression model among MLH showed ethnic differences in the predictive value of specific variables for most recent CD4 counts. Depression symptoms were a significant predictor for LA MLH only, contributing to the larger $R^{2}$ for this group relative to AA women. Also, the $t$-values for years since diagnosis were in opposite directions for LA and AA and may reflect differential HIV infection progression as a function of ethnicity. 
Such ethnicity effects are consistent with recent populationbased studies using similar biomarker composites showing increased health risk for male and female African Americans across all ages (Seeman et al. 2008) and for African American women specifically (Geronimus et al. 2006). Women and minorities have been identified as at high risk for health disparities in general, and for increased morbidity and mortality from HIV/AIDS specifically (Chu and Selwyn 2008). Thus, the relevance of ethnicity, medical variables (HIV status and associated disease and treatment factors), psychological symptoms and their interactions in relation to biomarker composites requires further study.

There are a number of limitations to the current research. First, the cross-sectional nature of the research design does not allow for examination of the biomarker index over time. Without longitudinal data, the temporal stability of the biomarker composite is unknown. This issue is particularly important if biomarker composites are to be used to evaluate quality of life, intervention efficacy, or even HIV-specific disease progression. Second, biological samples (urine and saliva) were not provided under supervision of research staff and thus could have been substituted with samples from non-participants. Nonetheless, unlike samples tested for substance use or infection, levels of the assayed biomarkers have no known social or tangible negative (or positive) consequence to the participant so the motivation for substitution is not apparent. Relatedly, recreational substance use and medical variables (e.g., CD4 count, viral load, medication compliance, and stage of HIV) were all based on self-report here. Whereas there is evidence that self-report shows a strong correlation with veridical outcomes for some variables (Cunningham et al. 2007), future studies would be strengthened by additional objective evidence (e.g., medical chart reviews or urine testing for substances) conducted close in time to biomarker assessment. Invasive blood draws to confirm CD4 and viral load, while increasing accuracy, could reduce participation rates and increase the cost of biomarker research. Future studies will require a careful balance of scientific priorities in addressing these issues. Third, assessment of psychosocial stress concurrent with biomarker collection was limited to brief instruments assessing depression, posttraumatic stress and global psychological distress symptoms. As a result, it is not possible to clarify in detail what psychological or socio-cultural aspects of daily life may have contributed to high biomarker composite indexes. Seeman et al. (2008) found that low education and income effects on biomarker composites are large, and remain significant even after control for age, gender, ethnicity and health behaviors like smoking. Further examination of biomarker composites in relation to such demographic factors, as well as the content of perceived stress (e.g., economic conditions, parenting challenges, racial/ethnic discrimination, intimate partner problems) and possible buffering factors (e.g., social support, effective coping strategies, etc.) will be needed to inform effective interventions.

Despite limitations, the present research demonstrates that relatively inexpensive and non-invasive biomarkers from home-collected urine and other simple health measures (height and weight, waist-to-hip ratio, blood pressure) can be obtained in community-based settings with minimal staff involvement. When combined into a composite index rather than analyzed in isolation, such biomarkers provide additional explanation of variance in predicting HIV relevant outcomes (e.g., CD4 counts). Biomarker composites created from the methods used here inherently select "extreme" scorers, thereby supporting comparisons of individuals at greatest risk and displaying the greatest "resilience" to HIV+ stress. Such a strategy may improve efforts to prevent and intervene among high risk minority women and their children.

Acknowledgments Research supported by NIMH grant R01 MH068194 and by UCLA GCRC grant \# M01-RR00865.

Open Access This article is distributed under the terms of the Creative Commons Attribution Noncommercial License which permits any noncommercial use, distribution, and reproduction in any medium, provided the original author(s) and source are credited.

\section{References}

Antoni, M. H. (2003). Stress management effects on psychological, endocrinological, and immune functioning in men with HIV infection: Empirical support for a psychoneuroimmunological model. Stress (Amsterdam, Netherlands), 6(3), 173-188. doi: 10.1080/1025389031000156727.

Antoni, M. H., Cruess, S., Cruess, D. G., Kumar, M., Lutgendorf, S., Ironson, G., et al. (2000). Cognitive-behavioral stress management reduces distress and 24-hour urinary free cortisol output among symptomatic HIV-infected gay men. Annals of Behavioral Medicine, 22(1), 29-37.

Antoni, M. H., Cruess, D. G., Klimas, N., Carrico, A. W., Maher, K., Cruess, S., et al. (2005). Increases in a marker of immune system reconstitution are predated by decreases in 24-h urinary cortisol output and depressed mood during a 10-week stress management intervention in symptomatic HIV-infected men. Journal of Psychosomatic Research, 58(1), 3-13.

Arendt, G. (2006). Affective disorders in patients with HIV infection: Impact of antiretroviral therapy. CNS Drugs, 20(6), 507-518. doi:10.2165/00023210-200620060-00005.

Beck, A. T., Steer, R. A., \& Garbin, M. G. (1988). Psychometric properties of the Beck Depression Inventory: Twenty-five years of evaluation. Clinical Psychology Review, 8, 77-100. doi: 10.1016/0272-7358(88)90050-5.

Boarts, J. M., Sledjeski, E. M., Bogart, L. M., \& Delahanty, D. L. (2006). The differential impact of PTSD and depression on HIV disease markers and adherence to HAART in people living with HIV. AIDS and Behavior, 10(3), 253-261. doi:10.1007/s10461006-9069-7. 
Brown, J. L., \& Vanable, P. A. (2008). Cognitive-behavioral stress management interventions for persons living with HIV: A review and critique of the literature. Annals of Behavioral Medicine, 35(1), 26-40.

Centers for Disease Control and Prevention (CDC). (2009). Death rate for HIV disease among women, by race and age group-United States, 1987-2005. Morbidity and Mortality Weekly Report, 58(11), 286.

Chander, G., Himelhoch, S., \& Moore, R. D. (2006). Substance abuse and psychiatric disorders in HIV positive patients: Epidemiology and impact on antiretroviral therapy. Drugs, 66(6), 769-789. doi: 10.2165/00003495-200666060-00004.

Chang, E., Sekhar, R., \& Patel, S. (2007). Dysregulated energy expenditure in HIV-infected patients: A mechanistic review. Clinical Infectious Diseases, 44(11), 1509-1517. doi:10.1086/ 517501.

Chittiprol, S., Kumar, A. M., Satishchandra, P., Shetty, K. T., Rao, R. S. B., Subbakrishna, D. K., et al. (2008). Progressive dysregulation of autonomic and HPA axis functions in HIV-1 clade C infection in South India. Psychoneuroendocrinology, 33(1), 3040. doi:10.1016/j.psyneuen.2007.09.006.

Chu, C., \& Selwyn, P. A. (2008). Current health disparities in HIV/ AIDS. The AIDS Reader, 18(3), 144-165.

Cole, S. W. (2008). Psychosocial influences on HIV-1 disease progression: Neural, endocrine, and virologic mechanisms. Psychosomatic Medicine, 70, 562-568. doi:10.1097/PSY.0b013 e3181773bbd.

Cole, S. W., Kemeny, M. E., Fahey, J. L., Zack, J. A., \& Naliboff, B. D. (2003). Psychological risk factors for HIV pathogenesis: Mediation by the autonomic nervous system. Biological Psychiatry, 54(12), 1444-1456.

Cole, S. W., Korin, Y. D., Fahey, J. L., \& Zack, J. A. (1998). Norepinephrine accelerates HIV replication via protein kinase Adependent effects on cytokine production. Journal of Immunology (Baltimore, MD: 1950), 161(2), 610-616.

Crepaz, N., Passin, W. F., Herbst, J. H., Rama, S. M., Malow, R. M., Purcell, D. W., et al. (2008). Meta-analysis of cognitivebehavioral interventions on HIV-positive persons' mental health and immune functioning. Health Psychology, 27(1), 4-14. doi: 10.1037/0278-6133.27.1.4.

Crimmins, E. M., Johnston, M., Hayward, M., \& Seeman, T. (2003). Age differences in allostatic load: An index of physiological dysregulation. Experimental Gerontology, 38(7), 731-734. doi: 10.1016/S0531-5565(03)00099-8.

Cruess, S., Antoni, M., Cruess, D., Fletcher, M. A., Ironson, G., Kumar, M., et al. (2000). Reductions in herpes simplex virus type 2 antibody titers after cognitive behavioral stress management and relationships with neuroendocrine function, relaxation skills, and social support in HIV-positive men. Psychosomatic Medicine, 62(6), 828-837.

Cunningham, C. O., Li, X., Ramsey, K., \& Sohler, N. L. (2007). A comparison of HIV health services utilization measures in a marginalized population-Self-report versus medical records. Medical Care, 45(3), 264-268. doi:10.1097/01.mlr.00002502 94.16240.2e.

Foa, E., Cashman, L., Jaycox, L., \& Perry, K. (1997). The validation of a self-report measure of posttraumatic stress disorder: The posttraumatic diagnostic scale. Psychological Assessment, 9, 445-451. doi:10.1037/1040-3590.9.4.445.

Gaynes, B. N., Pence, B. W., Eron, J. J., \& Miller, W. C. (2008). Prevalence and comorbidity of psychiatric diagnoses based on reference standard in an HIV plus patient population. Psychosomatic Medicine, 70(4), 505-511. doi:10.1097/PSY.0b013e31 $816 \mathrm{aa} 0 \mathrm{cc}$.

Geronimus, A. T., Hicken, M., Keene, D., \& Bound, J. (2006). "Weathering" and age patterns of allostatic load scores among blacks and whites in the United States. American Journal of Public Health, 96(5), 826-833. doi:10.2105/AJPH.2004.060749.

Glei, D. A., Goldman, N. Chuang, Y. L., \& Weinstein, N. (2007). Do chronic stressors lead to physiological dysregulation? Testing the theory of allostatic load. Psychosomatic Medicine, 69(8), 769776. doi:10.1097/PSY.0b013e318157cba6.

Glover, D. A., Gracia-Aracena, E. F., Rice, E., Lester, P., \& Rotherum-Borus, M. J. (2009). Salivary cortisol/DHEA ratios in HIV+ women. (Manuscript in preparation).

Glover, D. A., Gracia-Aracena, E. F., \& Mohlman, J. (2008). Peripheral biomarker composite associated with smaller hippocampal volume. NeuroReport, 19(13), 1313-1316. (Aug 27).

Glover, D. A., \& Poland, R. E. (2002). Urinary cortisol and catecholamines in mothers of child cancer survivors with and without PTSD. Psychoneuroendocrinology, 27(7), 805-819. doi: 10.1016/S0306-4530(01)00081-6.

Glover, D. A., \& Stuber, M. L. (2006). Allostatic load in PTSDsymptomatic middle-aged mothers of child cancer survivors. Psychiatry: Biological and Interpersonal Processes, 69(3), 191203. doi:10.1521/psyc.2006.69.3.191.

Hellhammer, J., Schlotz, W., Stone, A. A., Pirke, K. M., \& Hellhammer, D. (2004). Allostatic load, perceived stress and health: A prospective study in two age groups. Annals of the New York Academy of Sciences, 1032, 8-13. doi:10.1196/annals. 1314.002.

Ironson, G., Solomon, G. F., Balbin, E. G., O'Cleirigh, C., George, A., Kumar, M., et al. (2002). The Ironson-Woods Spirituality/ Religiousness Index is associated with long survival, health behaviors, less distress and low cortisol in people with HIV/ AIDS. Annals of Behavioral Medicine, 24, 34-48. doi:10.1207/ S15324796ABM2401_05.

Israelski, D. M., Prentiss, D. E., Lubega, S., Balmas, G., Garcia, P., Mulhammad, M., et al. (2007). Psychiatric co-morbidity in vulnerable populations receiving primary care for HIV/AIDS. AIDS Care-Psychological and Socio-medical aspects of AIDS/ HIV, 19(2), 220-225.

Karlamangla, A. S., Singer, B. H., McEwen, B. S., Rowe, J., \& Seeman, T. (2002). Allostatic load as a predictor of functional decline: MacArthur studies of successful aging. Journal of Clinical Epidemiology, 55, 696-710. doi:10.1016/S0895-4356 (02)00399-2.

Kemeny, M. E., \& Schedlowski, M. (2007). Understanding the interaction between psychosocial stress and immune-related diseases: A stepwise progression. Brain, Behavior, and Immunity, 21, 1009-1018. doi:10.1016/j.bbi.2007.07.010.

Kirschbaum, C., Kudielka, B. M., Gaab, J., Schommer, N. C., \& Hellhammer, D. H. (1999). Impact of gender, menstrual cycle phase, and oral contraceptives on the activity of the hypothalamuspituitary-adrenal axis. Psychosomatic Medicine, 61(2), 154-162.

Kopinsky, K. L., Stoff, D. M., \& Rausch, D. M. (2004). Workshop report: The effects of psychological variables on the progression of HIV-1 disease. Brain, Behavior, and Immunity, 18(3), 246261. doi:10.1016/j.bbi.2003.08.003.

Korte, S. M., Koolhaas, J. M., Wingfield, J. C., \& McEwen, B. S. (2005). The Darwinian concept of stress: Benefits of allostasis and costs of allostatic load and the trade-offs in health and disease. Neuroscience and Biobehavioral Reviews, 29(1), 3-38. doi:10.1016/j.neubiorev.2004.08.009.

Kraemer, H. C., Schultz, S. K., \& Arndt, S. (2002). Biomarkers in psychiatry: Methodological issues. American Journal of Geriatric Psychiatry, 10(6), 653-659.

Kudielka, B. M., \& Kirschbaum, C. (2005). Sex differences in HPA axis responses to stress: A review. Biological Psychology, 69(1), 113-132. doi:10.1016/j.biopsycho.2004.11.009.

Kumar, M., Kumar, A. M., Waldrop, D., Antoni, M. H., \& Eisdorfer, C. (2003). HIV-1 infection and its impact on the HPA axis, 
cytokines, and cognition. Stress (Amsterdam, Netherlands), 6(3), 167-172. doi:10.1080/10253890310001605376.

Kumar, M., Kumar, A. M., Waldrop, D., Antoni, M. H., Schneiderman, N., \& Eisdorfer, C. (2002). The HPA axis in HIV-1 infection. Journal of Acquired Immune Deficiency Syndromes, 31(S2), S89-S93.

Leserman, J. (2003). HIV disease progression: Depression, stress and possible mechanisms. Biological Psychiatry, 54, 295-306. doi: 10.1016/S0006-3223(03)00323-8.

McCain, N. L., Munjas, B. A., Munro, C. L., Elswick, R. K., Jr., Robins, J. L., \& Ferreira-Gonzalez, A. (2003). Effects of stress management on PNI-based outcomes in persons with HIV disease. Research in Nursing and Health, 26(2), 102-117. doi: 10.1002/nur.10074.

McEwen, B. S. (2004). Protection and damage from acute and chronic stress-Allostasis and allostatic overload and relevance to the pathophysiology of psychiatric disorders. Annals of the New York Academy of Sciences, 1032, 1-7. doi:10.1196/annals. 1314.001.

McEwen, B. S., \& Seeman, T. (1999). Protective and damaging effects of mediators of stress: Elaborating and testing the concepts of allostatis and allostatic load. Socioeconomic status and health in industrial nations: Social psychological and biological pathways. New York Academy of Science, 896, 30-47. doi:10.1111/j.1749-6632.1999.tb08103.x.

McEwen, B. S., \& Wingfield, J. C. (2003). The concept of allostasis in biology and biomedicine. Hormones and Behavior, 43(1), 2-15. doi:10.1016/S0018-506X(02)00024-7.

Myers, H. F., Wyatt, G. E., Loeb, T. B., Carmona, J. V., Warda, U., Longshore, D., et al. (2006). Severity of child sexual abuse, posttraumatic stress and risky sexual behaviors among HIV-positive women. AIDS and Behavior, 10(2), 191-199.

Pickering, T. (1996). Recommendations for the use of home (self) and ambulatory blood pressure monitoring. American Society of Hypertension Ad Hoc Panel. American Journal of Hypertension, 9(1), 1-11. doi:10.1016/0895-7061(95)00341-X.

Rotheram-Borus, M. J., Lee, M. B., \& Gwadz, M. (2001). An intervention for parents with AIDS and their adolescent children. American Journal of Public Health, 91(8), 1294-1302. doi: 10.2105/AJPH.91.8.1294.

Sapolsky, R. M., Romero, L. M., \& Munck, A. U. (2007). How do glucocorticoids influence stress responses? Integrating permissive, suppressive, stimulatory, and preparative actions. Endocrine Reviews, 21(1), 55-89. doi:10.1210/er.21.1.55.

Scott-Sheldon, L. A. J., Kalichman, S. C., Carey, M. P., \& Fielder, R. L. (2008). Stress management interventions for HIV+ adults: A meta-analysis of Randomized controlled trials, 1989 to 2006. Health Psychology, 27(2), 129-139. doi:10.1037/0278-6133. 27.2.129.

Seeman, T. E., McEwen, B. S., Rowe, J. W., \& Singer, B. H. (2001). Allostatic load as a marker of cumulative biological risk: MacArthur studies of successful aging. Proceedings of the National Academy of Sciences of the United States of America, 98(8), 4770-4775. doi:10.1073/pnas.081072698.

Seeman, T., Merkin, S. S., Crimmins, E., \& McEwen, B. S. (2008). Education, income and ethnic differences in cumulative biological risk profiles in a national sample of US adults: NHANES III (1988-1994). Social Science and Medicine, 66, 72-87. doi: 10.1016/j.socscimed.2007.08.027.

Seplaki, C. L., Goldman, N., \& Glei, D. (2005). A comparative analysis of measurement approaches for physiological dysregulation in an older population. Experimental Gerontology, 40(5), 438-449. doi:10.1016/j.exger.2005.03.002.

Sherman, A. C., Leszcz, M., Mosier, J., Burlingame, G. M., Cleary, T., \& Ulman, K. H. (2004). Group interventions for patients with cancer and HIV disease: Part II. Effects on immune, endocrine, and disease outcomes at different phases of illness. International Journal of Group Psychotherapy, 54(2), 203-233. doi:10.1521/ ijgp.54.2.203.40390.

Whetten, K., Lesserman, J., Lowe, K., Reif, S., \& Whetten, R. (2006). Prevalence of childhood sexual abuse and physical trauma in an HIV-positive sample from the deep south. American Journal of Public Health, 96(6), 1028-1030. doi:10.2105/AJPH.2005. 063263.

Whetten, K., Reif, S., \& Whetten, R. (2008). Trauma, mental health, distrust, and stigma among HIV-Positive persons: Implications for effective care. Psychosomatic Medicine, 70(5), 531-538. doi: 10.1097/PSY.0b013e31817749dc.

Yehuda, R., Teicher, M. H., Trestman, R. L., Levengood, R. A., \& Siever, L. J. (1996). Cortisol regulation in posttraumatic stress disorder and major depression: A chrono-biological analysis. Biological Psychiatry, 40(2), 79-88. doi:10.1016/0006-3223 (95)00451-3. 